\title{
The descending respiratory, pathway in man
}

\author{
P. W. NATHAN ${ }^{1}$ \\ From the National Hospital for Nervous Diseases, Queen Square, London
}

The descending respiratory pathway may be considered to be the most important tract of the spinal cord; yet its position in man is unknown. It is important to work out the location of these descending fibres not only to complete anatomical knowledge but also to provide those practising surgery on the cervical cord with essential information. The absence of this knowledge contributed to the death of some of our patients after cordotomy and this has also been a cause of death in patients from other series of cordotomies. Following Stookey's (1943) report of his experience of high cervical cordotomies, Penfield (1943) said: 'I have had a patient in whom I sectioned the pain tract at the second cervical segment under local anaesthesia. Suddenly, as the wound was being sutured, while he was talking and in the midst of a sentence, he died . . . Autopsy showed no explanation of it.' Stookey replied that he had never had such an experience following unilateral cordotomy but he had with the bilateral operation. He added: 'In bilateral cordotomy, I think that sectioning of the anterior lateral columns probably interferes with the higher respiratory pathway coming down from the medulla to the diaphragm ... The difficulties I have had have been with bilateral cordotomies and I felt they were respiratory deaths, due to interruption of an upper respiratory pathway.' Undoubtedly many neurosurgeons have had the same experience: patients having had unilateral and more commonly bilateral cordotomies in the cervical region may die suddenly, often in their sleep, and at necropsy no explanation has been forthcoming. It will be shown in this paper that an adequate high cervical cordotomy will damage most of the descending fibres controlling respiratory movements.

In 1830, Sir Charles Bell made the suggestion that the tract for controlling respiratory movements lies in the lateral part of the cord, somewhere between the anterior and posterior roots. Although since then there has been work on animals that confirms Sir Charles Bell's conjecture, there has been, as far as I can ascertain, no evidence produced for man.

In 1895, Porter looked for the location of the pathway running 'from the bulb to the phrenic 'Member external scientific staff, Medical Research Council. nuclei' in the dog and the rabbit. In a single experiment on the dog he cut through the cord at the first cervical segment, leaving intact 'a part of the lateral column' on one side; 'the dog continued to breathe, somewhat feebly'. In a rabbit he cut the right half of the cord at the second cervical segment; he then cut the left half of the medulla oblongata 'close behind the calamus scriptorius' to $1 \mathrm{~mm}$. from the lateral surface. Following a period of artificial respiration, the animal started to breathe again spontaneously. Dividing the remaining region of the medulla permanently stopped respiration. He concluded that 'the respiratory impulse descends in the lateral column.' Allen (1927) also carried out partial sections of the cord in the cat and in the guinea-pig. His main purpose was to find out whether a "visceral bulbospinal pathway' exists; he also made some observations on the pathway for impulses to the motor neurones of the diaphragm and intercostal muscles. He concluded that the medullospinal respiratory impulses were conducted chiefly in the ventral part of the lateral columns and in the lateral part of the anterior columns.' Tosatti (1939a, $1939 \mathrm{~b}$ ), who also carried out partial sections of the cord cranial to the phrenic nuclei in the dog and the rabbit, came to the conclusion that there are two pathways, one main tract and one reserve pathway that comes into action only when the main tract is blocked. The main tract runs in the lateral column and the reserve tract in the anterior column. Pitts (1940), also using the method of partial sections of the spinal cord in the cat, concluded that the respiratory pathway lies in the anterior column and in the anterior part of the lateral column; he found it to be mainly uncrossed. Gardner and Haddad (1953) found in the cat that breathing continues as long as 'but one ventrolateral region of the cord' is left intact, the rest of the cord being divided. Hukuhara, Nakayama, and Okada (1954) traced the descending fibres in the cat and the dog by seeking for the action potentials of the respiratory discharge that originates in the medulla oblongata; they traced the location of this discharge in the cervical cord to a region of the white matter lying very close to the grey matter, near the substantia intermedia lateralis. 
Thus the evidence obtained from dogs, rabbits, guinea-pigs, and cats shows that the descending fibres run in the anterior and/or the lateral columns of the cord.

\section{MATERIALS AND METHODS}

The material for this study consists of eight main cases and five confirmatory cases; in all of them the operation of antero-lateral cordotomy for the pain of cancer was performed in the cervical segments of the cord. In the eight main cases, full histological examination of the affected region in the cervical cords was carried out by Dr. Marion Smith. No post-mortem examination was possible in the five confirmatory cases; in them the position of the lesion made at operation could be deduced from the region of loss of pain sensibility caused by this operation.

For many years I was hoping that an opportunity would occur of studying the effect on respiratory movements of cutting the lateral cortico-spinal tract but as this operation is no longer performed, there seemed no point in waiting any longer for the chance of investigating such a case. Accordingly, Oliver's (1953) cases in which the lateral cortico-spinal tract was cut are quoted as control cases, for in them there was no disturbance of respiratory movements; in our cases of cordotomy these movements were seriously disturbed.

Before the first and representative case is given in detail, it is essential for the argument to take note of Pitts' (1940) findings in his work on the location of this pathway in the cat. He found that 'even with hemisection of the cord', there was always some return of ipsilateral respiratory movements within the survival period of 10 days. It is clear from Pitts' work that this recovery must be due to fibres descending in the opposite side of the cord; no other explanation seems to be possible. Similarly in the cases reported here, respiratory movements were never permanently stopped by the operation. It is thus clear that no long-lasting paralysis of respiratory movements on the side of the lesion is to be expected from cutting the relevant ipsilateral descending fibres: recovery from the paralysis does not mean that all the relevant ipsilateral fibres have not been divided.

\section{HISTOLOGICAL METHODS}

Transverse sections of the cord through the region of operation stained by a wide variety of methods, detailed in previous papers (Nathan and Smith, 1951, 1953), were examined to establish the exact location of the operative lesion. On the basis of the location and extent of the incision, the cases have been divided into groups; photographs of sections of the cords of four cases will be shown, each section typical of each group of cases.

\section{METHODS OF EXAMINING RESPIRATION}

Day-to-day observations were made on respiration by routine clinical methods. In addition the diaphragm was examined by radiological screening, the patient usually being in the upright position, and the excursion of the two halves of the diaphragm was measured on the screen; sometimes screening after the operation had to be done with the patient lying on his back. During screening after the operation, various procedures were carried out to try and induce respiration of the paralysed side. The activity of the intercostal muscles was examined electromyographically with co-axial needle electrodes, samples from the two sides being taken simultaneously. In five of the patients changes in respiration were recorded during the operation; in one patient volume changes of the right and left lungs were recorded independently by means of a volumetric recorder through a Carlen's endobronchial tube; in the other four, the movements were recorded mechanically.

\section{MAIN CASES}

Only one case will be presented in full detail.

CASE $84^{1}$ Before the operation of high cervical cordotomy for the pain due to a sarcoma of the right scapula, all respiratory movements were seen to be normal on clinical examination. Electromyographical recording from the intercostal muscles showed the presence of active units in phase with inspiration and expiration wherever they were looked for. Radiologically the excursions of the two halves of the diaphragm were seen to be of normal extent.

The operation was carried out on the left side of the cord, midway between the second and third cervical roots; the purpose was to obtain an upper level of analgesia as near to the level of the incision as possible and this necessitated making an incision that extendedoc far forwards and medially. This was successful and there resulted a detectable change in pain and thermal sensibility up to the distribution of the mandibular division of the fifth nerve, a loss of pain sensibility to a pin of $60 \mathrm{~g}$. weight up to the third cervical dermatome, and a loss of all pain and thermal sensibility up to the fifth cervical dermatome.

Illustrations from the respiratory records made during the operation are shown in Figure 1. The records from the left lung, the side of the cordotomy incision, are shown as the upper records, and those from the right lung as the lower records. Figure 1a is the control record, showing the tidal volume of the two lungs before the spinal cord was incised. An incision was first made into the spinal cord at the second cervical segment, the point of the knife being inserted into the cord posterior to the line of attachment of the denticulate ligament to a depth of $6 \mathrm{~mm}$. and then withdrawn through the anterior part of the lateral column. As can be seen from Fig. 1b, this incision did not affect respiration for more than a few seconds, the tidal volume remaining essentially unchanged: the average length of 20 excursions of the left record was $30 \mathrm{~mm}$. before and $29 \mathrm{~mm}$. after the incision, a change of $3.3 \%$, whereas that of the right record was $45 \mathrm{~mm}$. before and $42 \mathrm{~mm}$. afterwards, a change of $6.6 \%$.

${ }^{1}$ The case numbers are those used throughout the series of cordotomies reported on previously by Nathan and Smith $(1951,1953,1958)$. 


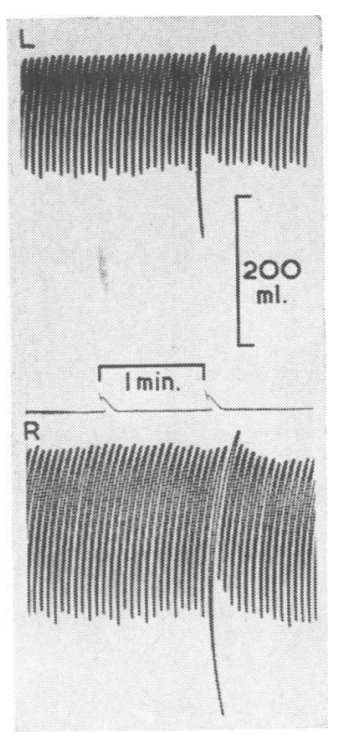

FIG. 1a


FIG. 1a. Control record of tidal volume; upper tracing of left lung, lower tracing of right lung.

FIG. 1b. Records made during two incisions into the left side of the cord at the second cervical segment; upper tracing of left lung; lower tracing of right lung.

FIG. $1 \mathrm{~b}$

From previous operations in the cervical segments, we knew that an incision not affecting respiratory movements would not give an adequately cranial level of analgesic zone; to bring the cranial extent of the analgesic zone higher up, the incision had to be more anterior. A second incision was then made, the knife being inserted to its full depth and being brought out as far medially and anteriorly as possible. This is shown in Fig. 1b, as 're-cut, incision enlarged'. Following this incision, the average excursion of the record on the left was $14 \mathrm{~mm}$., which is $47 \%$ of that before the incisions into the cord; the average excursion on the right was $43 \mathrm{~mm}$., which is $95 \%$ of that before the incisions. It was thus clear that the tidal volume had been considerably reduced on the left. It remained to ascertain if the volume changes on the left were in fact due to active respiratory movements of the left half of the chest and diaphragm or if they were due to movements transmitted from the right half of the chest. To determine this, the first manoeuvre was to constrict the tube between the apparatus and the right bronchus. This manoeuvre, designed to increase the negative pressure in the left side of the thorax, caused no increase in the excursions of the left side, and indicated that there was no mediastinal flap. To ascertain if any of the excursion of the left side was being transmitted from the movements of the right side, succinylcholine (Scoline) was then given intravenously. When all the active respiratory movements had been completely paralysed bilaterally, artificially induced respiratory pressure changes were applied to the right lung alone. It was then seen from the record that this artificially induced respiration gave a greater excursion of the right recorder than did the natural respiration, and this greater excursion was associated with only a small excursion on the left. It could be shown that when the right lung was being inflated artificially, the movement transmitted from the right caused the left to be deflated. It was shown that 4 to $5 \mathrm{~mm}$. of the excursion of the left side was due to movements transmitted from the right. Thus it followed that 14 minus $4 \mathrm{~mm}$. $(10 \mathrm{~mm}$.) was the true excursion on the left side.

It was concluded that the incision in the left side of the cord had greatly reduced the tidal air ipsilaterally; it did not stop all ipsilateral respiratory movements completely. It is interesting to note that whereas several observers agreed that no signs of respiratory movements could be detected clinically on the left, nevertheless about a third of the normal volume changes were still continuing.

When the patient was re-examined one hour after the incision in the cord had been made, almost no movements of the left side of the thorax were seen; there was a trace of movement visible antero-laterally in the most caudal part of the left hemithorax. Over the next hour the left side of the chest became very taut, becoming immobile in the inspiratory position.

Electromyographic sampling of the intercostal muscles of the two sides was proceeded with from one to seven hours after the incision into the cord. Before the recording was started, it was obvious that when the needles were inserted into the intercostal muscles, the shaft of the needle on the right moved with each respiratory excursion, whereas that on the left was motionless. Records taken during this period are shown in Figure 2. Extensive 

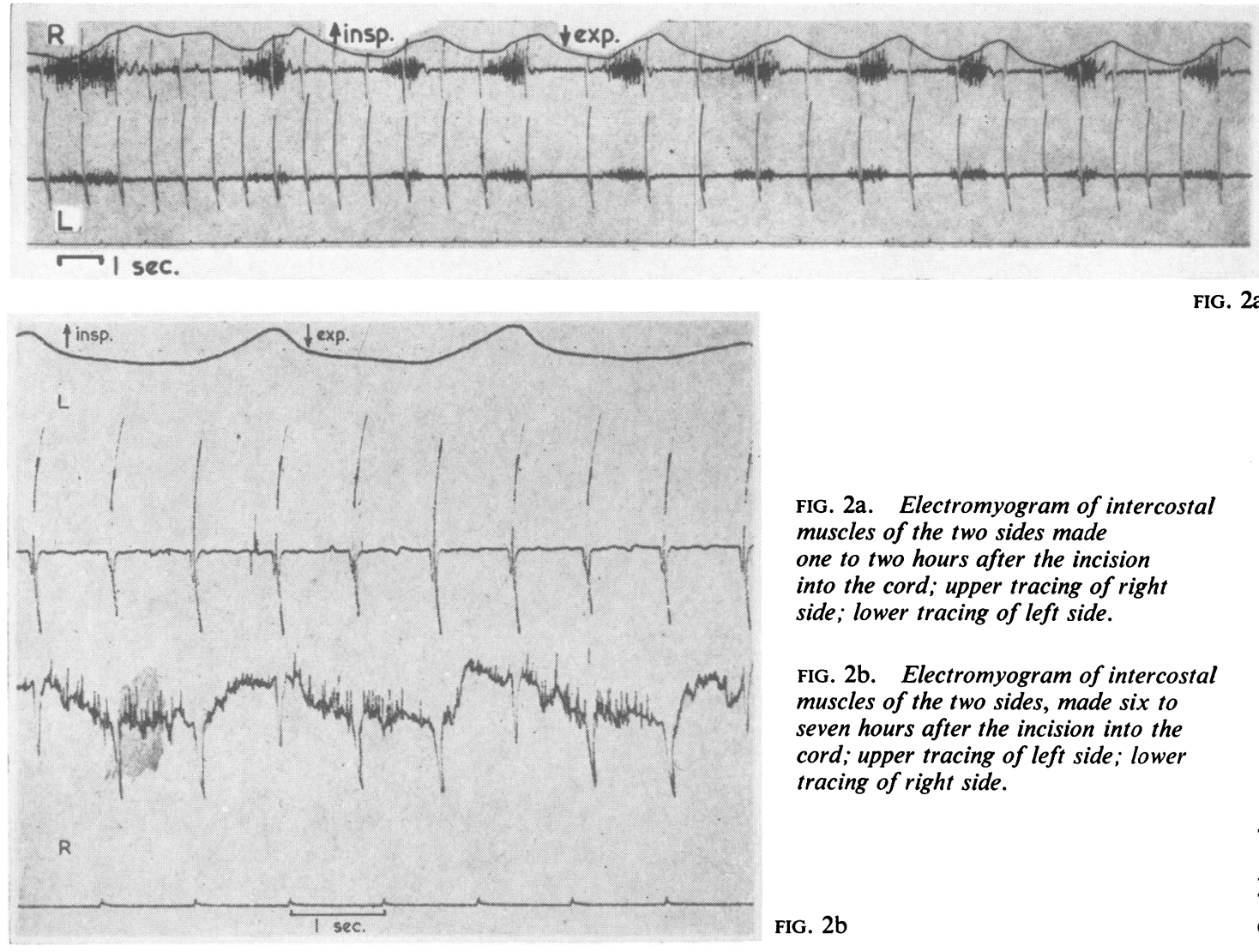

FIG. $2 \mathrm{a}$

FIG. 2a. Electromyogram of intercostal muscles of the two sides made one to two hours after the incision into the cord; upper tracing of right side; lower tracing of left side.

FIG. 2b. Electromyogram of intercostal muscles of the two sides, made six to seven hours after the incision into the cord; upper tracing of left side; lower tracing of right side.

FIG. $2 b$

sampling of the intercostal muscles on the left was needed before any active units in phase with respiration could be encountered; this was very different from the right side and from the left side before operation. This amount of activity, an example of which is shown in Fig. 2a, was insufficient, it will be noted, to cause enough movement of the left side of the thorax to be visible to any observer. Three to four hours after the incision into the cord, activity in other intercostal muscles on the left was again sought, but none was found. Six to seven hours after the incision into the cord, a further search was made; again no activity on the side ipsilateral to the lesion was found; this was repeated 30 hours after the incision into the cord, activity being looked for in the sixth and seventh intercostal muscles; none was found. A sample of the records made at this time is shown in Figure 2b. However, on this occasion some activity was found bilaterally in the parasternal region, the region where Taylor (1960) was usually able to find inspiratory activity.

By one week after the operation there was much restitution of respiratory movements of the left side; these movements were mainly abdominal, thus diaphragmatic. Three days later, when the patient was screened, it was observed that the movements of the left half of the diaphragm were occurring slightly later than the right on each respiratory movement and that the excursion was less on the left. For the next two weeks the movements of the left side of the chest, as judged clinically, remained far less than those of the right. On the twenty-first day after the operation the patient was given $5 \%$ carbon dioxide and $95 \%$ oxygen to breathe to see if the resulting hyperpnoea would increase the respiratory movements on the left to equal those on the right; it did not do so, although it greatly increased the rate and the extent of respiratory movements bilaterally. Oxygen lack, induced by rebreathing, the carbon dioxide being absorbed, had no effect on respiratory movements. Screening at this time, with the patient lying supine, showed normal excursion of the diaphragm on both sides. When the patient coughed or sniffed to order, there was a very slight delay of the movement of the left diaphragm compared to that of the right; the same delay was then observed when the patient took a deep breath in or out, to order. The excursion of the two halves of the diaphragm was measured with the patient standing. Before operation it had been $5 \mathrm{~cm}$. on the right, $3.5 \mathrm{~cm}$. on the left; after the operation it was $3.8 \mathrm{~cm}$. on the right, $1.9 \mathrm{~cm}$. on the left. Thus, although on the post-operative examination, the 
right was $76 \%$ of its previous value and the left was $54 \%$ of its previous value, the excursion of the left half of the diaphragm had been reduced to about a half of that of the right. Thirty-six days after the operation the two sides of the chest showed equal movements on clinical examination.

With regard to the state of motility of the limbs, it was observed that when the patient awoke from the general anaesthetic, he could not move the right limbs, and they were flaccid. A trace of movement on command returned seven hours after the making of the incision. The upper limb eventually recovered well; the lower limb recovered less well and less rapidly, walking never being normal again during the remaining 68 days of the patient's life.

In Fig. 3 are shown transverse sections through the region of the operation in this case. A cordotomy incision is not always made absolutely at right angles to the long axis of the spinal cord; and such was the case in this patient. The extent of the incision is thus shown best in three photographs, all from the second cervical segment. Figure $3 a$ is a section immediately cranial to the incision; the degeneration of the posterior root in the lateral part of the posterior column on the left is due to cutting of the root at operation and is not a part of the cordotomy incision. Figure $3 \mathrm{~b}$ is through the middle of the incision, and Fig. $3 \mathrm{c}$ is through the most caudal part of the incision. The lesion in this patient is the largest of the cordotomy incisions. It caused a great but not quite complete interruption of the respiratory movements ipsilaterally. This paresis of respiratory movements was not permanent; recovery ensued, although it was far from complete three weeks after the operation. As has been stated in the introduction to the case histories, the conclusion from the occurrence of recovery of ipsilateral respiratory movements is not that some of the descending fibres remain intact, for recovery occurs in the cat even after hemisections of the cord. From cases in which there was a prolonged disturbance of respiratory movements, it may then be concluded that at least the majority of the descending fibres are involved in the lesion. In this case, the fact that this large lesion did not stop ipsilateral respiratory movements completely shows that either there are some fibres used for respiratory movements that descend antero-medial or posterior to this lesion, or there are fibres used for this function which do not descend in the ipsilateral white columns of the cord.

The next three patients all died within six days of the operation, as a result of interference with this descending pathway. The lesions in the cord were large and similar to that shown in Figure 3. These cases are of use in demonstrating that a lesion involving this descending tract may lead to the



FIG. 3a. Marchi section immediately cranial to the region of operation in case 84. The degenerating fibres in the posterior column on the left are due not to the cordotomy but to cutting of a posterior root.

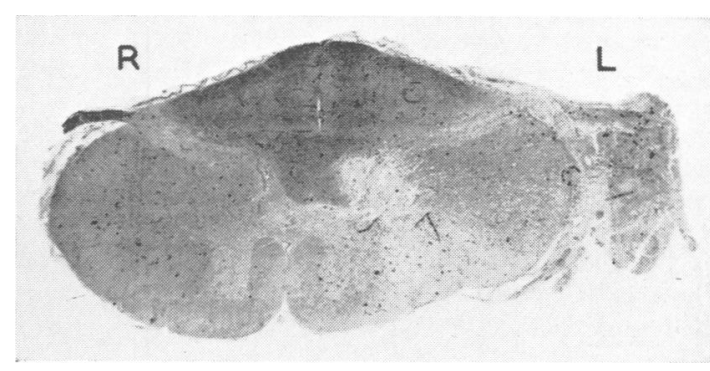

FIG. 3b. Haematoxylin and van Gieson section through the middle of the region of operation in case 84 .



FIG. 3c. Marchi section through the most caudal part of the region of operation in case 84. This incision involves the whole of the lateral column and a small part of the lateral part of the anterior column. 
patient's death, particularly when it is combined with carcinoma of the opposite lung.

CASE 64 Before the operation of high cervical cordotomy for the pain due to carcinoma of the left lung, the movements of the two sides of the chest were equal; however, screening of the chest showed that the left half of the diaphragm was paralysed; this was attributed to the carcinomatous tissue of the upper lobe of the left lung involving the left phrenic nerve. The operation was carried out on the right side of the cord between the second and third cervical segments. This caused loss of pain and thermal sensibility up to the distribution of the mandibular division of the fifth nerve. Immediately after the incision respiratory movements of the right side of the chest were very greatly diminished. The impression was gained that what little movement was still present on the right was a passive movement transmitted from the left side of the chest. On deep inspiration in the supine position no movement was seen of the upper abdominal wall on the right. After recovering from the general anaesthetic the patient could move the right limbs only slightly; active movements returned in the lower limb by 15 hours after the operation. Twenty-four hours after the operation active movements of the right side of the chest were still not seen. By 30 hours after the operation it appeared that diaphragmatic movements of the right side had returned, as judged by observation of the abdominal respiratory movements; but no activity was seen in the intercostal muscles on the right. The patient had become dyspnoeic and there was increased secretion of mucus in the bronchi and trachea. Signs of bronchopneumonia then developed, and 36 hours later the patient died.

The lesion in the cord was a large one, similar to that shown in Figure 3.

CASE 65 Before the operation of high cervical cordotomy for the pain due to carcinoma of the left lung, movements of the two sides of the chest were normal and screening showed that the two halves of the diaphragm moved normally. The operation was carried out on the right side of the cord at the second cervical segment. It caused a loss of pain and thermal sensibility, the upper level being high in the neck. Immediately after the incision into the cord, the respiratory movements of the right side of the chest ceased. Six hours after the incision into the cord, the right side of the chest did not move and the left moved inadequately. The patient could move all four limbs to command, the right slightly less than the left. Although he was placed in a respirator, he died 30 hours after the operation.

The lesion in the cord was a large one, similar to that shown in Figure 3.

CASE 42 Before the operation of high cervical cordotomy for the pain due to carcinoma of the left lung, the movements of the left side of the chest were diminished compared to those of the right. There was complete consolidation and collapse of the left lower lobe, the neoplasm obstructing the left lower bronchus. On screening, the left half of the diaphragm was shown to be raised and immobile. The operation was carried out on the right side of the cord between the first and second cervical segments. This caused a loss of pain and thermal sensibility below the sixth cervical dermatome. Immediately after the incision and for the following hour the patient was markedly cyanosed; there were no movements of the right side of the chest found on clinical examination and no activity of the intercostal muscles. Within three hours of the incision into the cord, slight respiratory movements had re-started ipsilaterally. These movements remained greatly diminished, until the patient died during his sleep five days after the operation.

This incision in the cervical cord caused no hemiparesis. The lesion in the cord was a large one, similar to that shown in Figure 3. Anteriorly it involved the anterior roots and posteriorly the anterior part of the lateral cortico-spinal tract.

CASE 44 Before the operation of high cervical cordotomy for the pain due to carcinoma of the left lung, there was diminution of the respiratory movements of the left side of the chest and considerable consolidation of the left lung. After the patient had been resting in bed for two hours, the respiratory rate was 18 per minute. The operation was carried out on the right side of the cord, the knife being inserted at the point of emergence of the first cervical root and carried anteriorly to the midline. It caused a loss of pain and thermal sensibility up to the distribution of the mandibular division of the fifth nerve. Immediately after this incision, respiratory movements of the right side were much diminished. On the following day the patient was cyanosed; both sides of the chest were moving, the right side far less than the left. Nineteen? days after the operation it was seen radiographically that movements of the two halves of the diaphragm were equal; they were less in amplitude than they had been before the operation.

This incision in the cervical cord caused no paresis or disturbance of motility of the limbs.

The lesion is shown in Fig. 4. It is one of the most anterior of the series. This lesion when compared to those of the cases discussed above is seen to be a small incision, and it does not involve the lateral cortico-spinal tract, nor the posterior part of the lateral column. It caused a great reduction of respiratory movements ipsilaterally. Recovery ensued, and the diaphragmatic movements were normal in $\mathbf{2 0}$ days. From the great diminution in the ipsilateral respiratory movements, it may be concluded that the majority of the descending fibres concerned with respiratory movements were involved in this lesion.

CASE 39 Before the operation of high cervical cordotomy for the pain due to carcinoma of the right lung, respiratory movement were equal on the two sides and both halves of the diaphragm showed normal excursions.

The operation was carried out on the left side of the cord in the more caudal part of the third cervical segment. 




FIG. 4. Marchi section through the region of operation in case 44. This incision involves the whole of the anterior column and the most anterior part of the lateral column.

It caused a loss of pain and thermal sensibility up to the third cervical dermatome. The record of chest movements made during operation showed that following the incision there were no respiratory movements of the left side. Twenty-four hours after the operation no respiratory movements were seen or felt on the left, and none were induced by re-breathing, anoxia produced by carbon dioxide being absorbed, nor by the administration of $5 \%$ carbon dioxide and $95 \%$ oxygen. By 48 hours after operation some respiratory movements had returned to the left side. Forty-eight hours later good and equal respiratory movements were recorded in the lower halves of both sides of the chest, although the movements of the left side were not quite as extensive as on the right. Fourteen days after the operation, screening of the diaphragm showed respiratory movements of both halves of the diaphragm, although the excursion of the right was far less than that of the left. When coughing and sniffing to command the patient could control expiratory and inspiratory movements normally and bilaterally. One week later, screening again showed less excursion of the left diaphragm, and it could be seen that movements of the left diaphragm were slightly delayed compared with those of the right; this was so, both for regular respiratory movements and for the movements induced by sudden sniffing. This examination was repeated two days later, the same results being obtained.

This operative incision in the cervical cord caused no actual paresis of the ipsilateral limbs, though the left lower limb felt subjectively heavier to move, and objectively it could be passively flopped more easily than the right when tone was examined.

The lesion in the cord was an anterior one, though less anterior than that shown in Fig. 4; it involved the most anterior part of the lateral cortico-spinal tract. It caused as much disturbance of ipsilateral respiratory activity as the large lesions of cases 84, 64, and 65, all of which involved a great part of this tract.

CASE 37 Before the operation of cervical cordotomy for the pain due to carcinoma of the ascending colon, the movements and excursions of the diaphragm were seen to be equal and normal and respiratory movements were equal on the two sides of the chest. The operation was carried out on the left side of the cord, the knife being inserted just anterior to the denticulate ligament at the level of the third cervical roots. It caused a loss of pain and thermal sensibility below the fifth cervical dermatome. Immediately after the incision, all respiratory movements of the left side of the chest had ceased and no breath sounds could be heard on the left, and none could be induced by the administration of $5 \%$ carbon dioxide, by oxygen lack, or on command to breathe deeply. Twelve hours after the operation there was no change but by 24 hours after the operation respiratory movements had returned on the left, although they were slighter in extent than those on the right. On the fourth day after the operation screening of the diaphragm showed equal and normal movements on both sides, and the movements of the chest wall had also returned to normal. Three weeks after the operation screening of the chest showed normal and equal movements of the diaphragm and of the chest walls. This incision into the cord caused no paresis or disturbance of movement of the ipsilateral limbs.

The lesion is shown in Fig. 5 and illustrates a lateral cordotomy incision. It caused a complete interruption of the respiratory movements ipsilaterally. Recovery ensued, so that the movements were normal in 20 days. It may be concluded that the majority of the descending fibres concerned with respiratory movements were involved in this lesion.

CASE 20 Before the operation of high cervical cordotomy for the pain due to carcinoma of the right lung, movements of the right side of the chest were found to be less than those of the left; this was most marked in the upper part; there was consolidation of the right upper lobe. The operation was carried out on the left side of the cord at the third cervical segment. It caused a loss of pain and thermal sensibility below the first thoracic dermatome. Immediately after the incision there were no movements of the left half of the chest. Within five

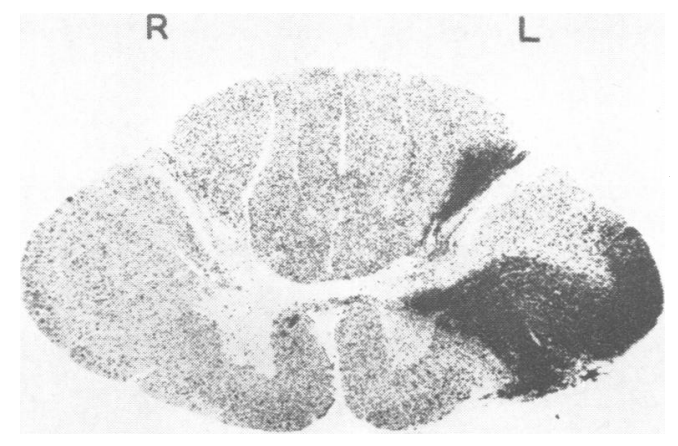

FIG. 5. Marchi section through the region of operation in case 37. This incision is restricted to the lateral column. 
days of the operation, respiratory movements of the left half of the chest had recovered to some extent, although on clinical examination they were still obviously less than those of the right; also the respiratory movements of the abdomen were less on the left. Three weeks after the operation, screening showed that the movements of both halves of the diaphragm were normal. This incision caused no paresis of the ipsilateral limbs.

The lesion in this case is shown in Figure 6. It was one of the smallest lesions of the series. As this lesion, involving only the most anterior part of the lateral cortico-spinal tract and not involving the anterior column, caused as much paralysis of ipsilateral respiratory movements as any of the other cases, it may be concluded that most of the descending fibres run in the region covered by this lesion.

\section{CORRELATION OF THE LESIONS IN THE MAIN CASES}

The large lesions, of which a typical example is shown in Fig. 3, involved the ipsilateral descending fibres concerned with respiratory movements. This lesion in fact consists of two incisions made into the cord: the first one was made in the posterior part of the lateral column, and had no effect on respiratory movements; the second one was made anteriorly, and involved respiratory movements. In support of the deduction from this case that it is the more anterior part of the region involved in Fig. 3 that is particularly important for the ipsilateral movements of respiration, the lesions shown in Figs. 4, 5, and 6 must be considered. The cases with the anterior and with the lateral lesions exhibited the same complete or all but complete paralysis of ipsilateral respiratory movements followed by recovery, as was shown by the cases with the large

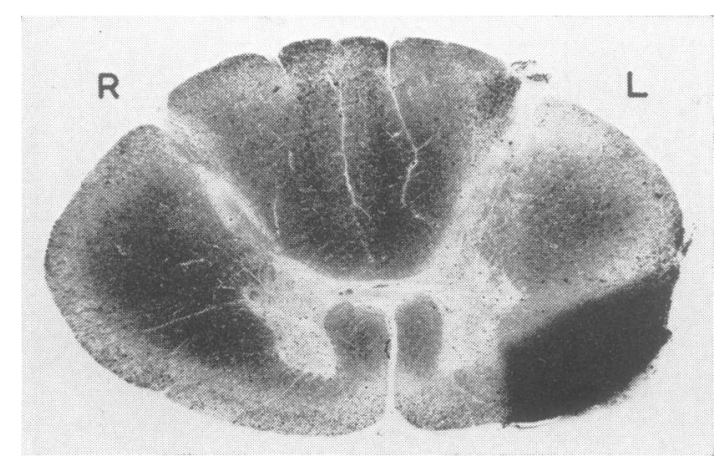

FIG. 6. Marchi section through the region of operation in case 20. This incision is the smallest of the series; it is restricted to the lateral column. lesions. It may be concluded that the fibres concerned are involved in the smaller lesions; they are situated in the anterior parts of the lateral column of the cord. Further, the lesion shown in Fig. 6 also involved all or most of the fibres. The region common to the lesions shown in Figs. 4 and 5 is shown in Fig. 7; this region was implicated in the lesion in all the cases with respiratory paralysis on the side of the lesion. It is concluded that it is within this area that most of the descending fibres concerned with respiration are located. This region of the cord is the most anterior part of the lateral column, that is, that region of the white matter lying immediately lateral to the emergence of the anterior roots.

As the lesions in some of the cases were large enough to cut through the anterior horn, it might be objected that such incisions damaged the cells of the anterior horn as well as involving these descending fibres. But such a lesion would in all cases have been cranial to the location of the cells
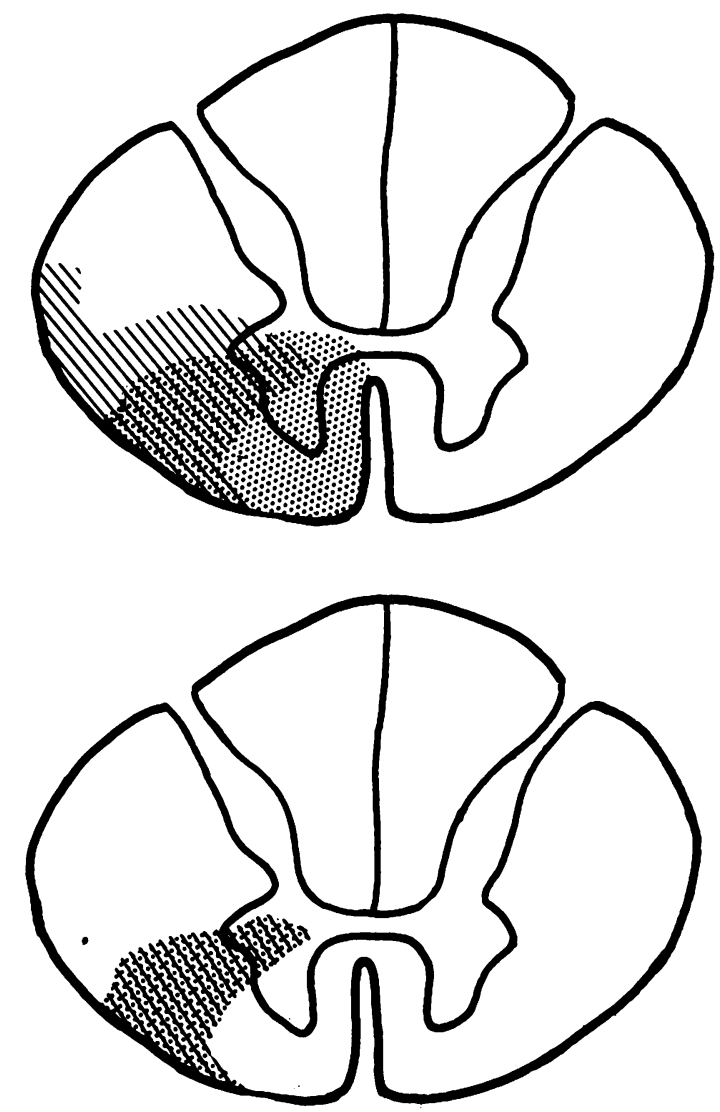

FIG. 7. Diagram of the two areas of cases 37 and 44 superimposed and of the area common to these two cases. 
innervating the diaphragm, and in most cases it would have been lateral to these cells, which are situated along the medial border of the anterior horn. The disturbance of respiration was not markedly different in the various cases, whether the lesion was away from or near to the fourth cervical segment and whether the lesion involved the grey matter of the anterior horn or not.

In all operations at least some of the fibres of the ipsilateral posterior root of the segment in which the incision was made were purposely divided or were damaged when the cord was rotated to enable the incision to be made. It has been shown by Nathan and Sears (1960) that cutting the fourth cervical posterior root causes a paralysis of the diaphragm persisting for weeks. It could be thought that the posterior rhizotomy performed in these cases might have had the same effect. This is unlikely, for in no case were the fourth cervical roots damaged; and further, the effects of the operation on respiration were no different whether the operation was performed at the first or the third cervical segments; and the operations also affected the intercostal muscles whereas a posterior rhizotomy at the third cervical segment could not have affected the motility of these muscles.

Similar incisions made at more caudal levels than the fourth cervical segment probably divide the fibres descending to the motor neurones of the intercostal muscles. But such cases have not been studied sufficiently to be reported upon.

None of the four patients with the smaller lesions had a hemiparesis; and none of these patients had the lateral cortico-spinal tract involved. It is thus apparent that a lesion causing paralysis or paresis of the ipsilateral respiratory movements does not necessarily cause hemiplegia or hemiparesis.

Unfortunately, although we have waited, the opportunity has never occurred of investigating a patient in whom an operation for the division of the lateral cortico-spinal tract in the cord was indicated. Oliver (1953) has carried out this operation in patients with Parkinsonism but by now this operation has been superseded by operations on the brain. Oliver was aware that division of the lateral cortico-spinal tract might cause disturbances of respiration. He found that breathing was not affected by the operation in four patients in whom bilateral pyramidotomy was performed in the cervical cord.

\section{CONFIRMATORY CASES}

If these descending fibres in the cervical cord are in the location that has been worked out, then the loss of pain sensibility associated with a lesion dividing these fibres will be throughout the cervical and upper thoracic segments. On the other hand, if there is a lesion posterior to the most anterior part of the lateral column where the descending fibres have been shown to run, then the loss of pain and thermal sensibility would be throughout the more caudal segments of the body, and might not include the upper thoracic and cervical segments. Further, a lesion made in the anterior part of the lateral column might well cause no disturbance of motility in the ipsilateral limbs, whereas a lesion made more posteriorly might involve the lateral cortico-spinal tract and might cause at least a temporary paresis of the limbs on the side of the incision.

Five of our cases of cordotomy, in which no postmortem confirmation of the lesion was obtained, can now be considered, to see how far this correlation of loss of sensibility and disturbance of respiration holds.

CASE 60 Before the operation of high cervical cordotomy for the pain due to a carcinoma of the breast invading the brachial plexus, the movements and excursions of the diaphragm were seen to be equal and normal and respiratory movements were equal on the two sides of the chest. The operation was performed on the right side of the cord at the second cervical segment. It caused a loss of pain and thermal sensibility up to the second cervical dermatome; it did not affect any form of sensibility below the level of the umbilicus. It caused paralysis of the ipsilateral diaphragm and the ipsilateral movements of the chest. It caused no paresis of the limbs; indeed, movements of the previously painful upper limbs were improved, doubtless due to the loss of pain.

CASE 99 Before the operation of high cervical cordotomy for the pain due to a carcinoma of the breast invading the brachial plexus, the movements and excursions of the diaphragm were seen to be equal and normal and respiratory movements were equal on the two sides of the chest. The operation was performed on the right side of the cord between the roots of the second and third cervical segments. It caused a loss of pain and thermal sensibility throughout the left side of the body below the third cervical segment. It caused no weakness of the limbs. The operation caused paralysis of the respiratory movements of the right side of the chest. There was a great reduction in the tidal air; after the incision the average respiratory rate was 24 per min., whereas before it had been 18. Forty-eight hours after the operation there were still no respiratory movements seen or felt on the right side of the chest, though the right side of the upper abdomen moved with respiration, albeit less than the left. By the sixth day after the operation respiratory movements were equal on the two sides of the chest and of the abdomen. One month after the operation screening showed equal movements of the two halves of the diaphragm.

CASE 100. Before the operation of high cervical cordotomy for the pain due to a carcinoma of the breast in- 
volving the brachial plexus, the left half of the diaphragm was found to be paralysed. The operation was performed on the right side of the cord at the second cervical segment. It caused a loss of pain and thermal sensibility throughout the left side of the body below the fourth cervical dermatome and a diminution in the second and third cervical dermatomes. It caused no change in the motility of the upper limb, but weakness in the lower limb, which recovered in a few weeks. The operation caused almost complete paralysis of the intercostal muscles on the side of the incision. The patient was cyanosed. Twenty-four hours after the operation it was possible to screen the patient; diaphragmatic movements were diminished on the side of the incision, the maximal excursion being $2.5 \mathrm{~cm}$. Slight movements of the right hemithorax were then seen; on auscultation air entry was found to be greatly diminished. The patient did not feel short of breath. By the sixth day after the cordotomy some cyanosis was still present but the movements of the right side of the chest had recovered to such an extent that they exceeded those of the left side.

CASE 102 Before the operation of high cervical cordotomy for the pain due to carcinoma of the bronchus, it was found that the movements of the right side of the diaphragm were much diminished, being less than $2.5 \mathrm{~cm}$. in extent when the patient was standing. The operation was performed on the left side of the cord at the first cervical dermatome. It caused a loss of pain and thermal sensibility throughout the right side of the body below the mandibular division of the fifth cranial nerve. Immediately after the operation the left limbs were weak but by seven days almost full power had returned to the upper limb and some power to the lower limb; six months after the operation no abnormality in the left limbs was found. The operation caused paralysis of ipsilateral respiratory movements; it could be shown that the little movement that was present was transmitted from the other side. Twenty-four hours after the operation there was some return of movements of the left half of the chest, not transmitted from the right; but it was apparent that at each breath the movement of the right side of the chest preceded that of the left. Forty-eight hours after the operation the patient complained of feeling short of breath, particularly after swallowing; he then had to make a conscious effort to breathe. There was still far less movement of the left chest than of the right and less movement on the left below the costal margin. Forty-eight hours later the two sides of the chest and the abdomen moved equally with respiration. Three weeks after the operation screening showed that the diaphragmatic movements on the left were normal in extent, and exceeded those of the right.

CASE 101 Before the operation of high cervical cordotomy for the pain due to carcinoma of the right bronchus the movements of the two sides of the chest were equal. The operation was performed on the left side of the cord between the roots of the second and third cervical segments. It caused a loss of pain and thermal sensibility throughout the right side of the body below the third cervical segment and a very slight weakness of the left upper limb. By a week after the operation the strength of the upper limb had recovered. The operation caused paralysis of the respiratory movements of the left half of the chest. Screening 24 hours after the operation showed no movements of the left half of the diaphragm. Recovery of the movements of the chest started within 48 hours of the operation.

\section{EVIDENCE FROM THE CONFIRMATORY CASES}

From our knowledge of the location of the fibres from the various segments of the body in the spinothalamic tract, and of the relation between damage to the lateral cortico-spinal tract and disturbances of motility of the limbs, it can be surmised where the lesions were in these five cases. In case 50, the lesion must have been restricted to the anterior column and to the most anterior part of the lateral column; in case 99 , the lesion must have been similar in location, and would have included the entire lateral column situated anterior to the denticulate ligament; in cases 100,102 , and 101 the lesion must have had the same anterior extent, and it must have extended further posteriorly, undoubtedly involving some of the lateral cortico-spinal tract. All these lesions involved the descending respiratory fibres; small anterior lesions involved them just as much as the large lesions, which involved the whole of the anterior column and also most of the lateral column.

From a purely anatomical point of view, it would 8 have been important to have had a lesion involving only the posterior part of the lateral column. But, as has been mentioned above, such lesions are no longer made.

\section{DISCUSSION}

It has been shown that a lesion involving the most anterior part of the lateral column of the cervical cord temporarily stops or greatly reduces ipsilateral respiratory movements. It is concluded that the majority of the descending fibres subserving the movements of respiration lie here. These descending fibres remain on the same side of the cord between the first cervical segment and their distribution to the ipsilateral motor neurones. Although a knife cut through this region causes a great diminution or a stopping of respiratory movements ipsilaterally, it may not be said that the integrity of these descending fibres is essential for respiratory movements of the diaphragm and intercostal muscles for recovery from the effects of this lesion soon ensues.

Although the evidence needs further confirmation, it seems clear that the fibres subserving respiratory movements do not descend in the cortico-spinal tract. This may be the reason why patients in the advanced stages of amyotrophic lateral sclerosis 
continue to live after they have lost all movements of the limbs.

Sir Charles Bell's surmise is seen to be correct: the fibres in man run in the same region as they were found to run in the dog, cat, guinea-pig, and rabbit, as shown by the work of Pitts (1940), Allen (1927), and Gardner and Haddad (1953). The results obtained in man after cordotomy are strikingly like those obtained by Pitts in the cat. He found that there was invariably some recovery during the 10day survival period of the experiments; and this was so when the cuts into the cord were so large that in fact they constituted hemisections.

It is true that it is common to find some recovery of function after most lesions in the central nervous system; but the amount of the recovery and its early onset are striking features of the recovery of respiratory movements following this lesion in the lateral column, as has been mentioned above. To account for it, the following possibilities may be considered. Pathways might be made available that are not normally used for respiratory movements. Or it might be that routes which had always been available but which were previously used only under conditions of great need might become used routinely. Or it might be that the trauma of cutting a large proportion of the descending fibres somehow has the effect of temporarily stopping conduction in other fibres that normally conduct in association with the cut fibres; eventually the remaining uninjured fibres might start to conduct impulses again. If fibres are used for the function of respiration that normally are not used for respiratory movements, this constitutes an example of vicarious functioning. If the sudden withdrawal of a usual source of excitation stopped conduction in pathways habitually used for this function, this would be an example of diaschisis, which is a transient state of diminished or abolished functioning of a part, not primarily involved by a lesion, being anatomically remote from it, but functionally related to the damaged part.

If alternative pathways are available it seems likely that they must be located contralaterally, for lesions as large as that shown in Fig. 3 can leave few fibres intact in the anterior column. If this is so, then each side of the cord has descending fibres potentially capable of innervating the respiratory muscles of both sides of the body. Tosatti found from his partial sections of the cord in the rabbit that the recovery of function depends on the existence of both crossed and uncrossed fibres in the anterior column. It is also possible that pathways in the grey matter, consisting of chains of neurones, might be available as alternative routes. Tosatti (1939a) was brought to the conclusion that there are two tracts available from the respiratory centre to the motor neurones of the phrenic nerve on each side of the cord: "una via "principale" decorrente nel cordone laterale del midollo cervicale ed une via "di reserva" decorrente nel cordone anteriore . . . la quale entra in funzione solamente quando siano state bloccata le vie principali decorrenti nei cordoni laterali'. $\mathrm{He}$ believes that the anterior tract contains both crossed and uncrossed fibres and that the lateral tract contains only crossed fibres. In a further study (1939b) he showed that the reserve tract is destined mainly for the motor neurones innervating the intercostal muscles and the motor neurones supplying those parts of the diaphragm innervated by the intercostal nerves.

The impression was gained from the cases studied here that the amount of respiratory activity during recovery on the side ipsilateral to the surgical lesion is to some extent related to the general level of excitation of central nervous activity and in particular to consciousness and the conscious awareness of the need to breathe. This statement is made in spite of the fact that induced oxygen lack or excess carbon dioxide in the inspired air did not induce ipsilateral respiration in the early stages after the operation. It was noticed that the respiratory activity returned intermittently, so that on one occasion intercostal muscle activity might be recorded, and on another occasion, particularly if the patient was tired or drowsy, none could be found. Two of the patients noted that they were breathing more, or more deeply, when they concentrated on their breathing; this voluntary attention to respiration helped an activity which is usually an automatic function. In other words, it seemed that the recovery of respiratory activity was to a certain extent related to the amount of excitation reaching the respiratory centre; and consciousness and effort contributed to the total level of excitability.

Some neurosurgeons perform bilateral high cervical cordotomies. In view of the importance of the descending respiratory fibres in this part of the cord, it is surprising that they are able to do this. Sometimes death does follow: for instance one surgeon reported that in two of his patients in whom the operation was performed in one stage, respiratory failure occurred during the second incision, and in both death ensued 24 hours later. In one the cordotomy was performed at the $\mathrm{C} 1$ and $\mathrm{C} 2$ segments, and in the other at the $\mathrm{C} 2$ and $\mathrm{C} 3$ segments. It seems that many surgeons do not usually make both incisions cranial to the fourth cervical segment, and so diaphragmatic breathing carries on unilaterally during the time respiratory movements are suspended on the other side. It is probable that at whatever level of the cervical cord the operation is performed, 
both incisions do not involve that region in which it has been shown that these descending fibres run. The reason for putting forward this suggestion is that in most cases a bilateral region of total analgesia is not obtained up to the segment of the incisions. As the fibres of the spino-thalamic tract issuing from the grey matter into the antero-lateral column lie near the region where this descending tract lies, an incision involving this tract is likely to cause immediate, complete analgesia up to the segmental level of the incision into the cord; and conversely, where there is not such analgesia up to the segmental level of the incision in the cord, this tract may well not be involved. Apart from such proffered suggestions why the fibres of the descending tract may not be involved bilaterally in high cervical cordotomies, it is possible that sometimes they are involved; on these occasions, the mechanisms giving rise to recovery of function, whatever they are, might be induced to come into play unusually early.

It has previously been reported by Nathan and Sears (1960) that cutting the posterior roots through which the phrenic nerve establishes afferent connexion with the spinal cord causes a temporary paralysis of the diaphragm. The recovery from the paralysis of the diaphragm occurs more quickly after cutting the descending fibres in the cord than it does after cutting the afferent nerves in the posterior root-which is a somewhat surprising finding.

The next question to be considered is whether the localization of the descending fibres as assessed according to function corresponds with the location of any recognized descending tract or system. The most important work on the descending tracts in this region comes from the Leiden school of neuroanatomists; most relevant are the theses by van Beusekom (1955), Busch (1961), and Staal (1961) in the cat and by Sie (1956) in man. Van Beusekom found that reticulospinal fibres are not united into compact tracts; there are no bundles consisting chiefly of reticulo-spinal fibres; the fibres lie everywhere in the anterior and antero-lateral columns. There was, however, 'a certain degree of concentration of reticulo-spinal fibres . . . between the medial longitudinal fascicle and the vestibulo-spinal tract, and within the medial longitudinal fascicle itself'. The area in which the descending fibres have here been shown to run in man is that where the vestibulospinal tract runs in the cat, as shown by van Beusekom and Staal; and this region contains many reticulo-spinal fibres. Busch and Staal divided the reticulo-spinal system of the cat into four groups: uncrossed medial reticulo-spinal fibres, originating in the caudal pons and rostral medulla, and descending in the anterior column; uncrossed lateral bulbospinal fibres (Thomas' fascicle) scattered in the anterior column largely outside the sulcomarginal zone; crossed ponto-spinal fibres descending in the posterior part of the lateral column near the rubrospinal tract; and crossed bulbo-spinal fibres descending contralaterally in the anterior column. Assuming that the arrangement in man is similar to that in the cat, then the region of the cord common to the lesions made here might include the uncrossed medial reticulo-spinal, the uncrossed lateral bulbospinal, and the crossed bulbo-spinal fibres. Sie found in man that the reticulo-spinal fibres do not form well-defined tracts; however, 'in the high cervical cord the majority of the ventral reticular fibre systems with a part of the dorsal reticular fibre system form the lateral part of the ventral funiculus'. He found that the reticulo-spinal fibres are completely intermingled with the fibres of the lateral vestibulo-spinal tract and of the medial longitudinal fascicle.

It will be noted that in the cat the reticulo-spinal fibres, except for those of the crossed ponto-spinal group, terminate along the medial border of the anterior horn. The motor neurones innervating the diaphragm are situated in the medial part of the anterior horn, as are those innervating the inter-o costal muscles, for this is the location of the motor neurones innervating all trunk muscles. It must bes stated, however, that most workers have found that the reticulo-spinal tracts continue throughout the length of the spinal cord; and so if it is with reticulo spinal fibres with which we are here concerned, only some of the fibres of this important system must be held to subserve the movements of the respiratory musculature. One other tract should be mentioned, the olivo-spinal tract. Although it is known that its fibres originate in the olive, it is possible that some may originate in the reticular substance near the olive, and these might include the neurones of the respiratory centre. This tract descends in the region where the evidence shows that the main descending pathway for the respiratory musculature runs; and the bulk of evidence concerning this tract shows that it does not descend further caudally than the fourth or fifth cervical segments.

It is of interest to consider the crossed phrenic phenomenon in relation to these cases. This phenomenon, as far as I can ascertain, has not yet been noted in man. In the cat a hemisection of the cord performed in the cervical region causes paralysis of the ipsilateral diaphragm. The contralateral phrenic nerve is then cut, causing paralysis of the contralateral diaphragm. This has the immediate effect of making the previously paralysed, ipsilateral diaphragm start working again.

This is not the place to discuss all aspects of this most interesting phenomenon. But as some of our 
patients had lesions, naturally occurring and surgically induced, which in effect approximated to the situation of the crossed phrenic phenomenon, it is of interest to consider the phenomenon as it affects man. It was found in the cat and the rabbit by Rosenblueth, Klopp, and Simeone (1938) that when a phrenic nerve was first cut and then the contralateral hemisection of the cord was carried out several weeks later, the hemisection did not then cause paralysis of the diaphragm on the side of the hemisection. A similar situation to this occurred in four of the patients reported here (cases $64,42,100$, and 102). In these four patients, the diaphragm on one side was paralysed before the cordotomy owing to involvement of the phrenic nerve in the neoplasm. The contralateral cordotomy did cause an immediate stoppage of respiratory movements in cases 64 and 42 and a great diminution of them in case 100 . In cases 64 and 42 some recovery from the paralysis of respiratory movements ensued; in these two it was insufficient to maintain life. In cases 100 and 102 recovery occurred, and a week after operation in case 100 and three weeks after operation in case 102 respiratory movements were better on the side of the cordotomy than on the side of the neoplasm.

It had already been found by Rosenblueth and Ortiz (1936) that species differ in whether they exhibit this phenomenon or not: dogs, cats, rabbits, and woodchucks show it; guinea-pigs and monkeys do not. It may now be stated that in the form of the phenomenon in which the phrenic nerve is first divided, man does not show the typical crossed phrenic phenomenon, for division of the descending fibres in the cord for innervation of the diaphragm does cause paralysis of the ipsilateral diaphragm.

The crossed phrenic phenomenon, the recovery of respiratory activity in all the patients reported here who lived long enough to show it, and the fact that the ipsilateral respiratory activity seemed to be influenced by the patient's efforts to breathe and the general level of neurological activity, these phenomena make one realize that neural routes are made available according to need. Pathways are opened up or used in relation to the total activity and needs of the animal; there is not one and only one route available; when the main or the usual pathway is divided, the impulses can reach the final common path via subsidiary, unusual, or new paths, which appear to be made available so that the necessary functional activity can be maintained.

\section{SUMMARY}

Cases of high cervical cordotomy were investigated to determine the effect of this operation on respiration. It was found that a lesion of the most anterior part of the lateral column of the first three cervical segments of the cord causes paralysis or a very great reduction in the ipsilateral respiratory movements. Recovery from the effects of this lesion ensues; within a few weeks of the lesion being made, respiratory movements become equal and normal on the two sides.

It is concluded that many or all of the fibres of the descending respiratory tract lie in this region; and that these fibres may form a part of the reticulospinal tract or they may run with the olivo-spinal tract.

I would like to thank Mr. Wylie McKissock and Drs. A. J. H. Hewer, T. A. Sears, and Marion C. Smith for the generous help they gave me, and Dr. E. A. Carmichael for the encouragement and the opportunities he gave me in carrying out this work.

\section{REFERENCES}

Allen, W. F. (1927). J. comp. Neurol., 42, 393.

Bell, C. (1830). The Nervous System of the Human Body. Longman, Rees, Orme, Brown, and Green, London.

Beusekom, G. T. van (1955). Fibre Analysis of the Anterior and Lateral Funiculi of the Cord in the Cat. Ijdo, Leiden.

Busch, H. F. M. (1961). An Anatomical Analysis of the White Matter in the Brain Stem of the Cat. Van Gorcum, Assen.

Hukuhara, T., Nakayama, S., and Okada, H. (1954). Jap. J. Physiol. 4, 145 .

Gardner, E., and Haddad, B. (1953). Amer. J. Physiol., 172, 475.

Nathan, P. W., and Sears, T. A. (1960). J. Neurol. Neurosurg. Psychiat. 23, 10.

$\longrightarrow$, and Smith M. C. (1951). Ibid., 14, 262.

- - (1953). Ibid., 16, 245.

(1958). Ibid., 21, 177.

Oliver, L. C. (1953). Parkinson's Disease and its Surgical Treatment. Lewis, London.

Penfield, W. (1943). Ass. Res. nerv. Dis. Proc., 23, 431.

Pitts, R. F. (1940). J. comp. Neurol., 72, 605.

Porter, W. T. (1895). J. Physiol. (Lond.), 17, 455.

Rosenblueth, A., and Ortiz, T. (1936). Amer. J. Physiol., 117, 495.

- Klopp, C. T, and Simeone, F. A. (1938). J. Neurophysiol., 1, 508.

Sie Pek Giok (1956). Localization of Fibre Systems within the White Matter of the Medulla Oblongata and the Cervical Cord in Man. Ijdo, Leiden.

Staal, A. (1961). Subcortical Projections on the Spinal Grey Matter of the Cat. Leiden.

Stookey, B. (1943). Ass. Res. nerv. Dis. Proc., 23, 416

Taylor, A. (1960). J. Physiol. (Lond.), 151, 390.

Tosatti, E. (1939a). Arch. Fisiol., 38, 533.

_- (1939b). Boll. Soc. ital. Biol. sper., 14, 615 and 677. 